\title{
3 Research S Suare e \\ Some Consideration About Mask Penetration: Influence of Material Properties, Particle Size and its Morphology As Well As Particle Physical Quantities
}

T. Ajtai ( $\nabla$ ajtai@titan.physx.u-szeged.hu )

University of Szeged

F. Kun-Szabó

University of Szeged

Sz. Hodovány

University of Szeged

G. Szabó

University of Szeged

Z. Bozóki

University of Szeged

\section{Research Article}

Keywords: KN95, FFP2, filtration efficiency, surgery mask, pandemic

Posted Date: January 24th, 2022

DOl: https://doi.org/10.21203/rs.3.rs-1276513/v1

License: (a) This work is licensed under a Creative Commons Attribution 4.0 International License. Read Full License 


\section{Abstract}

In this work composition, size, morphology and other physical quantities dependency of the most widespread face respirators and surgery masks are investigated using different aerosol sources in the submicron size domain. The airborne filtration efficiency of the sample materials are also demonstrated here. We have experimentally demonstrated high filtration efficiency of $\mathrm{NaCl}$, soot, DEHS and distilled water as well as airborne aerosols with limited size dependency. Using different mixing of distilled water and Sodium Lauryl Sulphate (SLS) surface tension dependency of filtration efficiency have been also quantified. It is experimentally demonstrated that the filtration efficiency is increased with increasing surface tension and the highest filtration efficiency have been realised with salvia equivalent surface tension value regardless of the sampled respirators or surgery mask. Finally, we also experimentally demonstrated that respirators has higher filtration efficiency than that of surgery mask and that the exhalation direction of the flow has negligible effect on filtration efficiency in all samples.

\section{Introduction}

Triggered at the end of 2018, the current coronavirus disease (COVID-19) caused by the SARS-CoV-2 virus has spread over the whole planet within months. As a response of COVID-19 pandemic many countries applied severe lockdown including physical and social distancing regulations recommended or enforced by their national health authorities and politics. The person to person transmission and touching the contaminated surfaces are defined as the primary transmission modes of viruses by The World Health Organisation (WHO) [1, 2]. The rapid spread of respiratory fluids and salvia including virus in distance of meters is also critical [3]. However, other alternative tracking such as airborne transition can also play an important role in virus spread too [4, 5]. However, despite of its importance, the corresponding details of transition methods and efficiency as well as the efficiency of respiratory and surgery mask usage are not completely defined yet $[5,6]$.

The respiratory droplet transition have a typical diameter of $5 \mu \mathrm{m}$ or larger [7]. In this regime, the transition tracking is defined dominantly by the fluid dynamics. However, the lifetime of such particles in the atmosphere is short. Therefore, besides the direct inhalation, the transition occur via deposition of virus containing respiratory fluids on surfaces is dominant in this regime as well [8]. In airborne transition, the virus can be transported by itself with its characteristic diameters of about $200 \mathrm{~nm}$. The airborne tracking of that virus sized aerosol sample driven mainly by diffusion. Alternatively, it can also be transferred by attachment of solid or embedded in liquid particulates dispersed in the air [9]. In this regime the particle tracking is driven by pure diffusion or fluid dynamics or in other complicated ways of those. The regime of airborne transition of virus is typically below $5 \mu \mathrm{m}$ [8]. However, the transport phenomena, the dynamics and the lifetimes of virus containing airborne particles may also depend on the size sub ranges inside that regime. In airborne transition the submicron region is also critical, not only due to its pandemic relevance but also the diverse health effect of air pollution too.

For prevention of COVID-19 transmission, besides physical distancing and hand hygiene, wearing face mask is suggested and enforced by the national health authorities and political governments in many countries. The surgery mask certified by different test methods like European standard EN 14683 and the filter face respirators certified according to test procedure of EN 149 (FFP respirators series) and §84.171 (KN respirator series). Those are primarily prescribed to wear for health workers. For general public usage, simple fabric mask with questionable filtration efficiency is at least demands in most countries [10]. However, mainly, because of the firm belief in its higher filtration efficiency and after the massive demands of medical mask or face respirators in the first few months of pandemic period caused shortage of supply of such devices, more and more surgery masks and face respirators have been appearing in the street too. Moreover, the resident of many countries wear surgery or face respirators or even fabric 
masks because of the poor air quality in their countries to protect themselves from adverse health effects related to air pollution too $[11,12]$. It is also worth to note, that presumably because of the mask wearing, the number of medical contact associated to lung diseases has dramatically decreased in COVID-19 pandemic period. In airborne particle assembly, the major health relevant composite is the soot (IPCC, 2007). Although the soot of airborne particle is not dominant in mass, but in number it is responsible for almost all air quality relevant adverse health effects [13]. The soot originating from the incomplete combustion of fossil fuels having versatile physicochemical properties. The soot having characteristic diameter below submicron size domain with fractal morphology even have one week lifetime in the atmosphere [14]. Due its small characteristic size, high adsorption capabilities for toxic substances and effective surface area, the soot can penetrate through even in alveolus and get directly into blood too. Since the deposition map of the lungs is strongly depends on the size and morphology (especially below $\mu \mathrm{m}$ size domain) the investigation of size, morphology, composition and other physical parameter dependency of mask penetration is critical issue in the context of its airborne filtration efficiency as well.

Removal of aerosol from an airstream is mainly driven by five different physical mechanism including gravitational settling, internal impaction, interception, diffusion and electrostatic attraction [15]. They are all shows size dependency. The first two is more efficient in particle settling towards the smaller size, while, the latter three shows increasing efficiency with increasing size. Besides all, the test protocols for surgery or face respirators $[16,17$, and 18$]$ do not specify the size dependency of filtration but cumulative efficiency. They are also use atypical aerosol standards such as $\mathrm{NaCl}$ or paraffin oil for test protocols as well as atypical measurement condition such as constant flow with high flow rate which is far from the realistic breathing conditions. Since the penetration could also depend on other physical quantities such as viscosity, surface tension, morphology or phase of particles, the more detailed investigation of filtration efficiency even in a sub micrometre size domain is actual issues having even pandemic or more comprehensive interest todays.

In this work we demonstrate the comprehensive, description type measurement results of size dependent filtration efficiency of three widely used mask or face respirators including surgery mask, FFP2 and KN95 respirators in the submicron size domain. Using different type of aerosol standards, the phase, the morphology and the surface tension effect on size dependent filtration efficiency are also investigated. Finally, the results of size dependent airborne filtration efficiency is also demonstrated here.

\section{Experimental Set-up, Methods And Sample}

Three simple experimental configurations for comprehensive investigation of three different type of mask samples were used in this work (Fig. 1). The set-up presented schematically in Fig. 1a adopted two type of liquid nebuliser successively. For generating $\mathrm{NaCl}$ and water aerosol standards TOPAS ATM 220 liquid nebuliser was used, while for producing monodisperse liquid aerosol in the higher size range condensation based technique based on the Sinclar Lamer principle was applied (model: MAG3000, Palas, $\mathrm{GmbH}$ ). In this procedure, $\mathrm{NaCl}$ aerosol was used to initiate the DEHS (Di-Ethil-Hexil-Sebacate) condensation onto its surface resulted in monodisperse aerosol assembly in the most relevant accumulation mode of the atmosphere. In this configuration diffusion dryer was used to stabilise the yield of particles. For generating soot fractal aggregates spark discharge generator (model: DNP2000, Palas, GmbH) was applied (Fig. 1b).

In this configuration two ejector diluter were implemented into the set-up to avoid the excess of concentration limit defined by the SMPS. For the investigation of airborne relevance of filtration efficiency ambient aerosol was used which entered the room through open window of the laboratory (Fig. 1.c). The aerosol standards were drawn through the sample fixed onto a cylindrical flange. The characteristic parameter of the flange were ID=40 $\mathrm{mm}$ and length of 200 
$m m$ respectively. The flow was maintained by the SMPS pump ensuring the atmospheric pressure condition in this configuration.

The number concentration and the size distribution of the emitted particle assembly were measured by a Scanning Mobility Particle Sizer (SMPS, TSI Inc., model: 3938) The SMPS consist of two parts. A differential mobility analyser (DMA) which is responsible for the size separation of the sampled particle assembly (DMA, model 3081, with Kr85 neutraliser model 3077). The Condensation Particle Counter (CPC, model 3752) is responsible to count the separated particles in this configuration. The sheath and aerosol flow rates were set to 2.3 and 0.23 litre per minute, thus the SMPS measured the size distribution over the range of $16-930,6 \mathrm{~nm}$ with resolution of $\pm 0.5 \mathrm{~nm}$ at $16 \mathrm{~nm}$ to $\pm 30 \mathrm{~nm}$ at $930 \mathrm{~nm}$. In order to minimize the shielding artefact, coinciding correction of the measured data was also applied. This technique provides mobility particle diameter $\left(d_{p}=d_{m o b}\right)$. This parameter set was used throughout this work. Both in the Fig. 1a and Fig. 1b configurations around 8 litre per minute aerosol flow were configured. Filtration efficiency of the sample materials were determined by measuring the transmission throughout the respective sample. The transition function of the flange without sampling material using different aerosol sources and its characteristic parameters including Count Median Diameter (CMD), Geometric Standard Deviation (GSD) and Total Number Concentration (TNC) are shown and summarised in Fig. 2. and Table 1. The aerosol standards using this study is including $\mathrm{NaCl}$, distilled water, DEHS, soot and surface tension modified distilled water using Sodium Lauryl Sulphate (SLS).

Table 1

characteristic parameters of emitted aerosol standards drawn through the empty flange.

\begin{tabular}{|lllllll|}
\hline & Dist.water & Soot & NaCl & 100 ppm SLS & 200 ppm SLS & MAG 3000 \\
\hline TNC & $4,8 \mathrm{E}+09$ & $4,6 \mathrm{E}+09$ & $2,4 \mathrm{E}+09$ & $9,2 \mathrm{E}+08$ & $7,3 \mathrm{E}+08$ & $1,4 \mathrm{E}+09$ \\
\hline GMD & 48,7 & 70,3 & 46,1 & 56,2 & 62,3 & 321,4 \\
\hline GSD & 60 & 90,3 & 56 & 89,3 & 99,4 & 341,2 \\
\hline
\end{tabular}

Three different samples were investigated including KN95 and FFP2 face respirators defined by §84.170 and EN 149 patents as well as a surgery mask using clinical circumstances defined by EN 14683. From all mask and respirator materials fitted area was cut, prepared and implemented onto the flange. In order to minimize the mask material uncertainty each measurement was repeated three times using freshly mounted different samples from the same mask type for all aerosol standards. Overall, the measurement cycle was the following: 30 minutes measurement of flange transition (6 scan in SMPS), than 30 minutes measurement of filter transition, finally 30 minutes measurement of flange transition. These are repeated at three times of each freshly mounted sample. As the measurement uncertainty 1 -sigma standard deviation of the average was calculated.

\section{Results And Discussion}

The size distribution and its characteristic parameters measured with empty flange and with filter material in both inhalation directions (in and exhalation) using different aerosol sources are shown and summarised in Fig. 3 to 6 . and Table 2 respectively. The related filtration efficiency as a function of particle diameter deduced from the measured data are also presented in those figures (left side). 
Table 2

characteristic parameters of emitted aerosol standards through the different mask types (both sides of masks)

\begin{tabular}{|c|c|c|c|}
\hline GMD & GSD & TNC & $\begin{array}{l}\text { Cumulative } \\
\text { filtration } \\
\text { efficiency } \\
\text { (\%) }\end{array}$ \\
\hline
\end{tabular}

(\%)

Cumulative filtration efficiency

(\%)

\section{Surgery mask $+\mathrm{NaCl}$}

Empty

flange

Inhalation $\quad 47,4 \quad 49,1 \quad 5,59 E+06 \quad 2,1$

$\begin{array}{llll}\text { Exhalation } \quad 46,1 \quad 45,7 & 4,58 \mathrm{E}+06 \quad 1,7\end{array}$

FFP2+NaCl

Empty

flange

Inhalation $52 \quad 43,2 \quad 2,94 \mathrm{E}+05 \quad 0,1$

Exhalation $\quad 51,7 \quad 43,6 \quad 5,04 \mathrm{E}+05 \quad 0,2$

$\mathrm{KN} 95+\mathrm{NaCl}$

Empty $\quad 46,4 \quad 55,4 \quad 2,72 E+08$

flange

Inhalation

47,7

Exhalation 49

49

44

Surgery mask+soot

Empty

70,3

flange

Inhalation $\quad 46,6 \quad 47,8 \quad 5,18 \mathrm{E}+07 \quad 1,1$

Exhalation $\quad 57,1 \quad 71,1$

$6,15 \mathrm{E}+07 \quad 1,4$

FFP2+soot

Empty

flange

Inhalation $\quad 55,6 \quad 47,5 \quad 2,21 \mathrm{E}+06 \quad 0,4$

peak_1

Inhalation $\quad 142,7 \quad 159,1$

peak_2

$\begin{array}{lllll}\text { Exhalation } & 55,3 & 47,6 & 9,69 \mathrm{E}+05 & 0,2 \\ \begin{array}{l}\text { KN95+soot } \\ \text { Empty } \\ \text { flange }\end{array} & 83,4 & 109 & 4,93 \mathrm{E}+08\end{array}$

Surgery mask+DEHS

Empty $\quad 328,5 \quad 75,9 \quad 1,19 E+09$

flange

Inhalation $\quad 317,9 \quad 82 \quad 1,28 E+07 \quad 1,1$

$\begin{array}{llll}\text { Exhalation } \quad 321,4 & 79,7 & 6,49 \mathrm{E}+06 & 0,5\end{array}$

FFP2+DEHS

Empty $\quad 323,2 \quad 83,3 \quad 1,28 \mathrm{E}+09$

flange

Inhalation $\quad 195,1 \quad 118,1 \quad 1,02 E+05 \quad 0,008$

peak_1

Inhalation $\quad 321,4 \quad 109,8$

peak_2

$\begin{array}{lllll}\text { Exhalation } & 312,7 & 88 & 3,87 \mathrm{E}+05 & 0,03\end{array}$

peak_1

Exhalation $\quad 271,2 \quad 295,5$

peak_2

\section{KN95+DEHS}

Empty $\quad 307,6 \quad 81,2 \quad 1,44 E+09$

flange

Inhalation $\quad 297,7 \quad 86,6 \quad 7,53 E+05 \quad 0,05$

Exhalation $\quad 292,8 \quad 77,3 \quad 9,05 E+05 \quad 0,06$
Surgery mask+distilled water

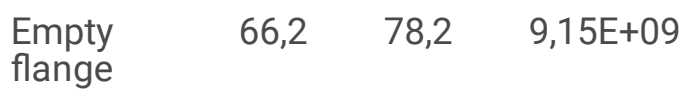

flange

$\begin{array}{llll}\text { Inhalation } \quad 53,8 \quad 52,2 & 8,99 \mathrm{E}+07 \quad 1\end{array}$

Exhalation $\quad 49,2 \quad 45,6 \quad 6,44 E+07 \quad 0,7$

FFP2+distilled water

Empty

$52,6 \quad 46$

$4,77 \mathrm{E}+09$

flange

Inhalation $\quad 48,7 \quad 60 \quad 7,85 E+06 \quad 0,2$

Exhalation $40 \quad 30,1 \quad 7,37 \mathrm{E}+06 \quad 0,2$

KN95+distilled water 


\begin{tabular}{|c|c|c|c|c|c|c|c|c|c|}
\hline & GMD & GSD & TNC & $\begin{array}{l}\text { Cumulative } \\
\text { filtration } \\
\text { efficiency } \\
\text { (\%) }\end{array}$ & & GMD & GSD & TNC & $\begin{array}{l}\text { Cumulative } \\
\text { filtration } \\
\text { efficiency } \\
(\%)\end{array}$ \\
\hline Inhalation & 105,6 & 230,1 & $8,04 \mathrm{E}+05$ & 0,2 & $\begin{array}{l}\text { Empty } \\
\text { flange }\end{array}$ & 55,6 & 66,7 & $5,85 \mathrm{E}+09$ & \\
\hline \multirow[t]{2}{*}{ Exhalation } & 142 & 174,5 & $1,16 \mathrm{E}+05$ & 0,02 & Inhalation & 49 & 42 & $1,05 \mathrm{E}+07$ & 0,2 \\
\hline & & & & & Exhalation & 50,3 & 44,4 & $1,05 E+07$ & 0,2 \\
\hline \multicolumn{5}{|c|}{ Surgery mask+ambient aerosol } & \multicolumn{5}{|c|}{ FFP2+ambient aerosol } \\
\hline \multicolumn{3}{|l|}{$\begin{array}{l}\text { Empty } \\
\text { flange }\end{array}$} & \multicolumn{2}{|l|}{$1,30 \mathrm{E}+05$} & \multicolumn{3}{|l|}{$\begin{array}{l}\text { Empty } \\
\text { flange }\end{array}$} & \multicolumn{2}{|l|}{$1,30 \mathrm{E}+05$} \\
\hline \multicolumn{3}{|l|}{ Inhalation } & $5,35 E+02$ & 0,4 & \multicolumn{3}{|l|}{ Inhalation } & $8,00 E+02$ & 0,6 \\
\hline \multicolumn{10}{|c|}{ KN95+ambient aerosol } \\
\hline \multicolumn{3}{|l|}{$\begin{array}{l}\text { Empty } \\
\text { flange }\end{array}$} & $1,30 \mathrm{E}+05$ & & & & & & \\
\hline \multicolumn{3}{|l|}{ Inhalation } & $8,00 E+02$ & 0,4 & & & & & \\
\hline
\end{tabular}

The $\mathrm{NaCl}$ is the dominant aerosol standard for verifying the filtration efficiency $[16,17]$. The size distribution of empty flange and flange with filter material both in and exhalation directions using $\mathrm{NaCl}$ aerosol shows significant filtration efficiency regardless of the type of mask and direction of inhalation throughout the emitted particle size range (Fig. 4.). Take into account the two order of differences in transmission (relative to the empty flange transmission) moderate size dependency of filtration efficiency have been found in all samples (Fig. 3). The maximum cumulative filtration efficiency of $6 \%$ and $5 \%$ are defined in FFP2 and KN95 respirators. In the dominant size range in number-concentration (below about 200nm) the FFP2 and KN95 respirators shows negligible differences in transmission $(<0.2 \%)$ regardless in the flow direction. Although at the higher size of the particles $(>200 \mathrm{~nm})$ the differences are increased it is remained below $1 \%$ regardless of the direction of flow. However, it is worth to note that above $200 \mathrm{~nm}$ the number concentration of the emitted particulate is below $1 \%$ (zoomset of Fig. 3.). The surgery mask shows smaller but also significant filtration efficiency without negligible size dependence. The filtration efficiency is below or about $6 \%$ even in the higher size range. The cumulative filtration efficiency of surgery mask is below $2 \%$, which is much better than the defined value of the respiratory protocols. The deduced transition function of empty and sample material implemented flange in bot flow direction shows also high efficient filtration when spark discharge generated soot particle assembly is used (Fig. 3.). Smaller than $2 \%$ cumulative filtration efficiency have been realized in the whole emitted size domain regardless of the type of mask or flow directions. However, KN95 respirator shows significant differences in flow direction resulted in U-shape curve (in both flow directions) (Fig. 3). Nearly one order of magnitude higher filtration efficiency has been realised in exhalation than in the reversed flow direction. Although this deviation is significant the efficiency of soot filtration is remarkable and fulfil the patent requirement not only in cumulative aspect but also in any size sequent even in both flow directions. The FFP2 sample shows high similarity in cumulative efficiency of filtration and also that of size dependency resulted in a similar U-shape (below 3\%). However, in exhalation flow direction bimodal size distribution have been measured in FFP2 mask sample. Due to its unexpected measurement results, the whole measurement cycle was repeated at three additional freshly mounted sample, but provides the same distribution curves in all measurement cycle. The surgery mask sample provides also high efficient filtration of soot particles having cumulative filtration efficiency below $3 \%$, but with decreasing tendency towards the higher sizes with the minimum of about 200-300 $\mathrm{nm}$ size domain. 
The $\mathrm{NaCl}$ particles have a compact morphology with sharp lamella-like boundary conditions, while the soot has a morphology of fractal aggregates including spherical primary particles with irregular spatially arrangement [14]. The differences in morphology in the overlapping characteristic size domain makes possible to investigate the morphology dependence of the filtration efficiency. From the measurement data one can conclude that morphology cannot affect the filtration efficiency of the investigated mask sample in the $20 \mathrm{~nm}$ to about $600 \mathrm{~nm}$ size domain. Moreover, since the combustion generated soot fractal aggregates are responsible many divers health effects, the high efficiency in soot filtration highlight the advances of mask wearing even over the pandemic period too. The liquid phase filtration efficiency were modelled with DEHS aerosol condensed onto a salt core particle and distilled water aerosol assembly. The size distribution of transmission and the size dependent filtration efficiency of DEHS are shown in Fig. 4.

Using DEHS particle emission all the investigated sample provides high efficient filtration throughout the emitted size domain for all type of masks and for both flow directions. In all configurations less than $1 \%$ of transmission efficiency was realised with this aerosol. It is also provides distribution of transmission modelled with bimodal distribution function in inhalation direction in case of KN95 sample. However, it also worth to note, that due to the extremely high filtration efficiency, it is not affect anyhow the filtration behaviour of the sample. Surgery mask provides also remarkable, but worse filtration efficiency for DEHS aerosol too (Table.2). Some microphysical properties of the respiratory fluids and salvia were modelled at first approximation with distilled water aerosol generated by liquid nebuliser (Fig. 4). Using distilled water aerosol high efficient filtration efficiency was realised too. Both respirators provide less than $1 \%$ transition throughout the emitted size domain, while surgery mask provides less than $2 \%$ cumulative filtration efficiency with worst value of that about $5 \%$ at around $200 \mathrm{~nm}$ size. Using water aerosol source we have also realised measurable differences in flow direction in some cases. However, besides high efficient filtration of water, it is not affected the quality of the investigated materials anyway. The surface tension of salvia is differ from that of distilled water. Therefore to investigate the surface tension affect for filtration efficiency and for approximate the measurement condition to more realistic circumstances emulsion of distilled water and Sodium Laurate Sulphate (SLS) was created at two different mixing ration (100 ppm and $200 \mathrm{ppm}$ SLS). The salvia equivalent surface tension was realised by 200 ppm SLS mixing rate. The cumulative filtration efficiency of distilled water (ST=71.99 mN/m), $100 \mathrm{ppm}$ $(\mathrm{ST}=65,33 \mathrm{mN} / \mathrm{m})$ and $200 \mathrm{ppm}$ SLS $(\mathrm{ST}=48.93 \mathrm{mN} / \mathrm{m})$ mixing are shown in Fig. 5. The surface tension dependency of cumulative filtration efficiency is clearly shown that with increasing surface tension the filtration efficiency is increased. The highest filtration efficiency is realised at 100 ppm and 200 ppm SLS sources which is more or less is the same as surface tension of salvia (ST 60 $\mathrm{mN} / \mathrm{m})$ [19].

Finally, ambient aerosol sources was also used to mimic the airborne transition of mask samples. The size of the sampled airborne particles fall between $10 \mathrm{~nm}$ and $950 \mathrm{~nm}$ diameter. The measurement results is shown high filtration efficiency for all type of mask and flow directions (Fig. 6). The cumulative filtration efficiency of less than $1 \%$ have been realised with moderable size dependency throughout the whole emitted size range in all samples.

\section{Summary And Conclusion}

The respective transition of number concentration and the size distribution of different aerosol sources covered the submicron size domain were measured at three different widely used respirators such as FFP2 and KN95 and surgery mask defined by EN 149, §84.171 and EN 14683 respectively. The ex and inhalation direction of flow effect on transition was also investigated. Finally, airborne aerosol sources was used to measure the filtration efficiency in the air quality relevant submicron size domain.

Applying different sources makes possible to investigate the size, phase, surface tension and viscosity dependency of filtration even in the overlapped size domain. Using $\mathrm{NaCl}$ particles which is the most relevant aerosol standards for quantifying filtration efficiency and the spark discharge generated soot aerosol allow the investigation of morphology

Page $7 / 14$ 
dependency of filtration. The compact sharped layer bounded $\mathrm{NaCl}$ aerosol provides the similar filtration efficiency than that of soot particles having fractal-like morphology regardless of the mask types and flow directions. Therefore, one can conclude, that the morphology of the filtered aerosol is not affected the filtration efficiency in this regime. It is experimentally demonstrated that liquid phase aerosol sources having different microphysical properties provides different filtration efficiency. The distilled water aerosol source was used to model more realistic physical parameters of respiratory fluids or salvia. All the investigated mask or respiratory materials shows high efficiency of filtration for water aerosol in the investigated size domain. Although liquid aerosols having different viscosity were tested in this study, the correlation between the viscosity and filtration efficiency was not realised. However, the measurement of particles transition generated from distilled water and SLS mixing at different SLS mixing rates shows definite surface tension dependency on filtration. The higher filtration efficiency have been reached with 100 ppm and 200 ppm SLS concentration which is well modelled the real surface tension condition of salvia $(\sim 60 \mathrm{mN} / \mathrm{m})$. It is also experimentally demonstrated here that although the different aerosol sources have spread from around $10 \mathrm{~nm}$ to $950 \mathrm{~nm}$ size domain with overlapping subdomains the size dependency of filtration efficiency for the whole size range cannot be quantified in an additive way, because of the significant, phase, composition and other microphysical dependency of those. Finally, both the artificially generated soot and the ambient transition measurement have also verified experimentally that both the respirators and the surgery mask are also a useful tool for airborne relevant health diseases protection and the usage of those goes beyond those pandemic relevance. Overall, the filtration efficiency of the surgery and face respirators is depends strongly not only the composition of the aerosol standards but its phase and other microphysical parameters too. Therefore, the pandemic relevance of its usage quantified by the modelled aerosol standards and measurement protocols are needs more detailed investigations. However, all type of mask provides remarkable filtration of efficiency of the airborne particles in the health relevant submicron size region. Therefore, the usage of face respirators or surgery masks in the ambient having poor air quality is useful for protection of health diseases associated to air pollution too.

\section{Declarations}

\section{Acknowledgements}

This work was supported by the TKP2020 (project NKFIH-1279-2/2020); the Hungarian Scientific Research Foundation (project OTKA NKFI-138176).

\section{Data availability}

The data supporting the articles is available on request at the correponding author.

\section{Competing interests}

The authors declare no competing interests.

\section{Author contributions}

T. Ajtai: responsible for the scientific side of the project. Organizing and control measurement and the data evaluations

F. Kun-Szabó: built up the measurement set-up, operating the instruments, data evaluation according to the given protocols

Sz. Hodovány: built up the measurement set-up, operating the instruments, data evaluation according to the given protocols 
G. Szabó: Institute leader. Providing the financing side of the project. Scientific advisor of the project. Participating the data interpretation and writing the MS.

Z. Bozóki: Group leader. Providing the financing side of the project. Scientific advisor of the project. Participating the data interpretation and writing the MS.

\section{References}

[1] WHO (World Health Organization). 2020a. Country \& technical guidance - Coronavirus disease (COVID-19). Geneva, Switzerland: World Health Organization. Accessed July 03, 2020.

[2] WHO (World Health Organization). 2020c. Coronavirus disease 2019 (COVID-19): Situation Report - 66, March 26, 2020. Geneva, Switzerland: World Health Organization. Accessed July 10, 2020.

[3] Liu, S., and A. Novoselac. 2014. Transport of airborne particles from an unobstructed cough jet. Aerosol Sci. Technol. 48 (11):1183-94. doi:10.1080/02786826.2014.968655

[4] Zhang, R., Y. Li, A. L. Zhang, Y. Wang, and M. J. Molina. 2020. Identifying airborne transmission as the dominant route for the spread of COVID-19. Proc. Natl. Acad. Sci. USA. 117 (26):14857-63. doi:10.1073/pnas.2009637117.

[5] Morawska, L., and D. K. Milton. 2020. It is time to address airborne transmission of COVID-19. Clin. Infect. Dis. doi:10.1093/cid/ciaa939.

[6] Klompas, M., M. A. Baker, and C. Rhee. 2020. Airborne transmission of SARS-CoV-2: Theoretical considerations and available evidence. J. Am. Med. Assoc. 324 (5):441-442. doi:10.1001/jama.2020.12458.

[7] Kutter, J. S., M. I. Spronken, P. L. Fraaij, R. A. M. Fouchier, and S. Herfst. 2018. Transmission routes of respiratory viruses among humans. Curr. Opin. Virol. 28:142-51. doi:10.1016/j.coviro.2018.01.001.

[8] WHO (World Health Organization). 2014. Infection prevention and control of epidemic- and pandemic-prone acute respiratory infections in health care. Geneva, Switzerland: World Health Organization. Accessed July 03, 2020.

[9] Tsuda, A., Henry, F. S., \& Butler, J. P. (2013). Particle transport and deposition: basic physics of particle kinetics. Comprehensive Physiology, 3(4), 1437.

[10] Drewnick, F., Pikmann, J., Fachinger, F., Moormann, L., Sprang, F., \& Borrmann, S. (2021). Aerosol filtration efficiency of household materials for homemade face masks: Influence of material properties, particle size, particle electrical charge, face velocity, and leaks. Aerosol Science and Technology, 55(1), 63-79.

[11] Jacobson, M. Z. 2012. Air pollution and global warming: History, science, and solutions. New York: Cambridge University Press.

[12] WHO (World Health Organization). 2016. Ambient air pollution: a global assessment of exposure and burden of disease. Geneva, Switzerland: World Health Organization. Accessed July 03, 2020.

[13] Pope III, C. A., \& Dockery, D. W. (1999). Epidemiology of particle effects. In Air pollution and health (pp. 673-705). Academic Press.

[14] Andreae, M. O., \& Gelencsér, A. (2006). Black carbon or brown carbon? The nature of light-absorbing carbonaceous aerosols. Atmospheric Chemistry and Physics, 6(10), 3131-3148. 
[15] Hinds, W. C. 1999. Aerosol technology - Properties, behavior, and measurement of airborne particles. 2nd ed. New York: Wiley Interscience, John Wiley \& Sons.

[16] EN14683. 2019. Medical face masks - Requirements and test methods. German version EN 14683:2019+ AC:2019. DIN Deutsches Institut für Normung e.V. Berlin: Beuth Verlag GmbH.

[17] 149:2001 a1 Respiratory protective devices. Filtering half masks to protect against particles. Requirements, testing, marking

[18] BS EN 14683:2019 Medical face masks. Requirements and test methods

[19] Niraula, T. P., Shah, S. K., Chatterjee, S. K., \& Bhattarai, A. (2018). Effect of methanol on the surface tension and viscosity of sodiumdodecyl sulfate (SDS) in aqueous medium at 298.15-323.15 K. Karbala International Journal of Modern Science, 4(1), 26-34.

\section{Figures}

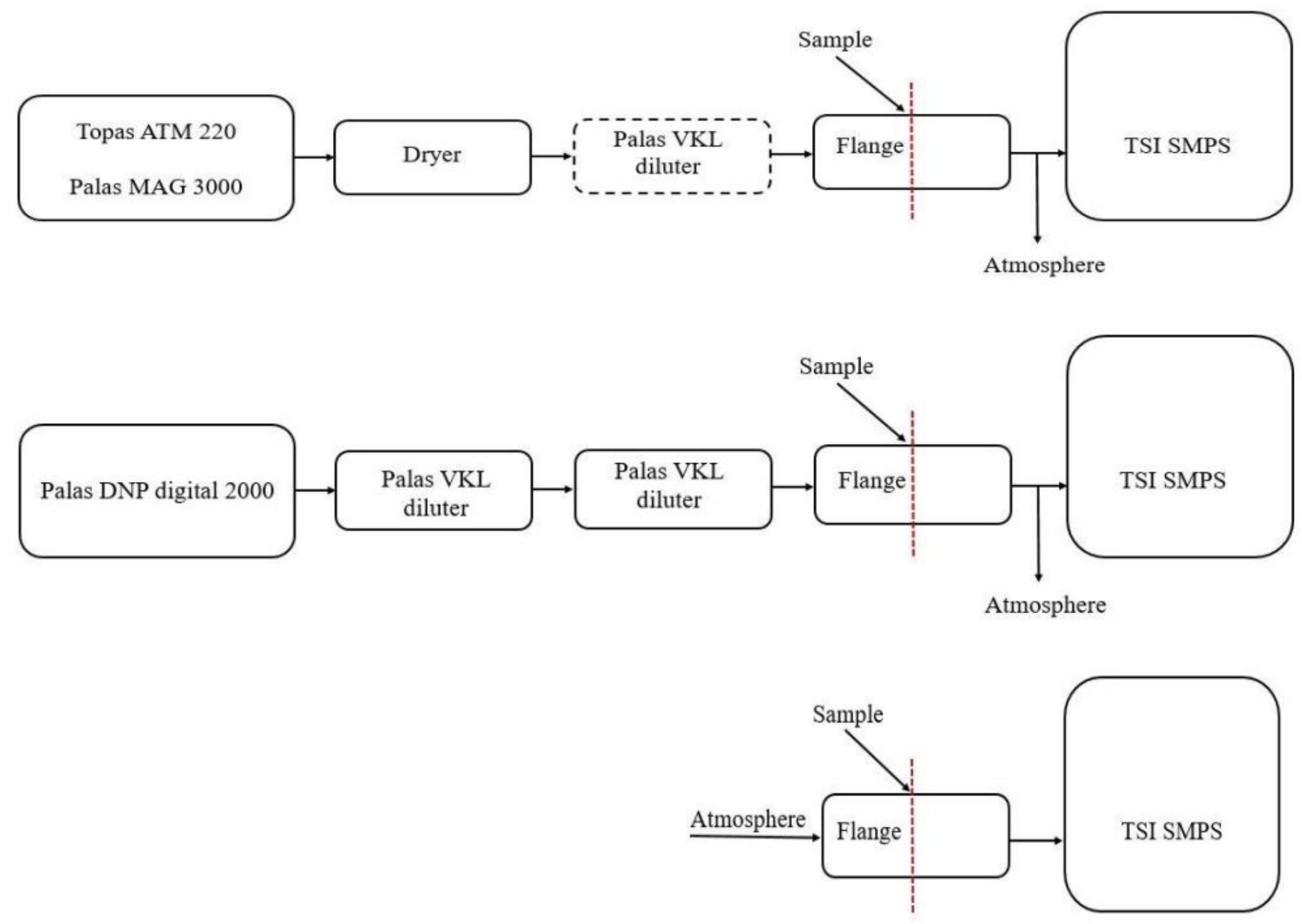

Figure 1 
Experimental set-up for filtration efficiency measurement of different sample materials: (a) the experimental set up using two type of liquid nebulizer to generate, $\mathrm{NaCl}$, DEHS, distilled water and paraffin oil aerosols, (b) experimental setup for generating spark discharged soot aerosol assembly, (c) experimental set up for ambient relevance measurement of filtration efficiency.

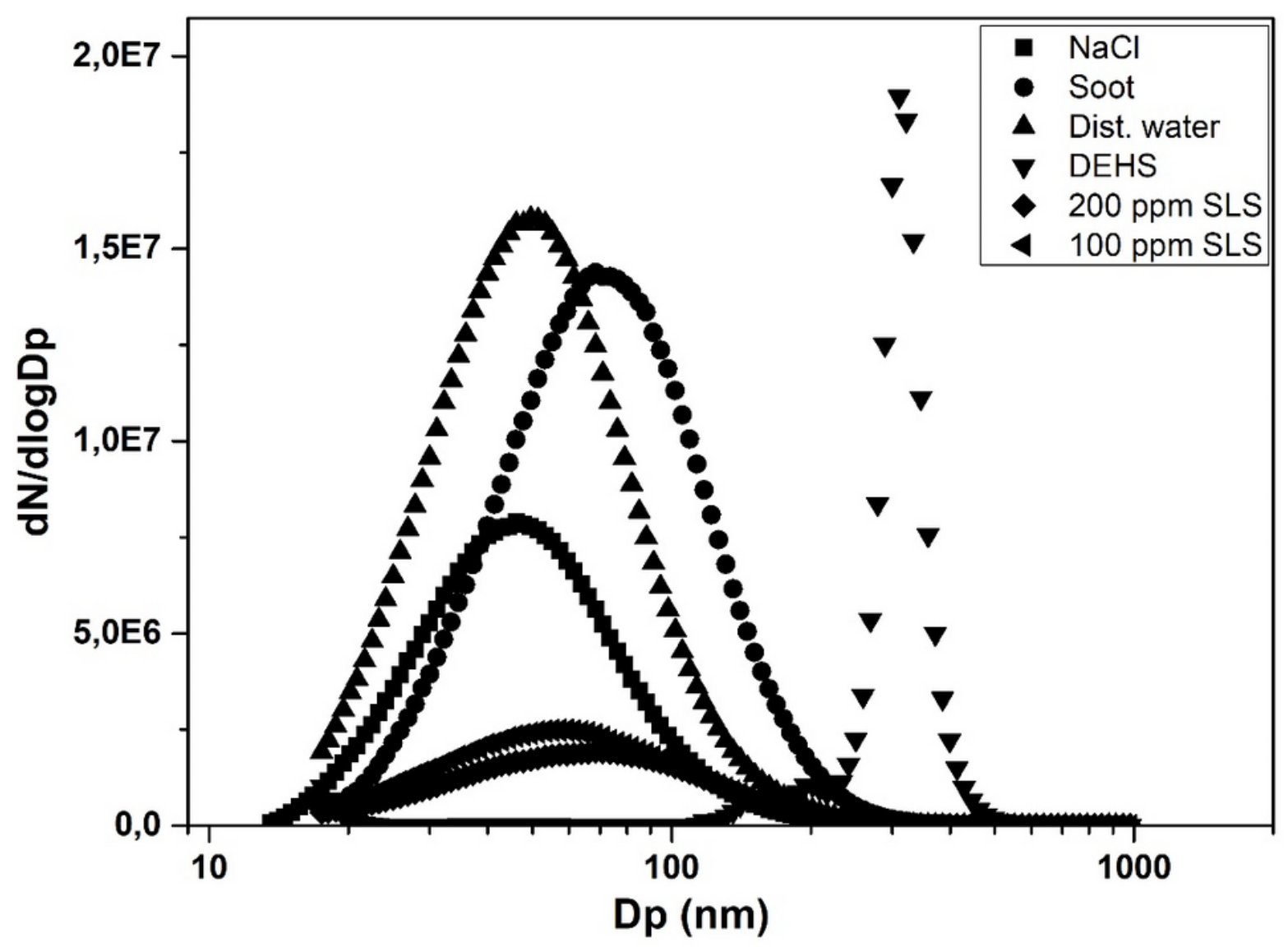

Figure 2

Size distribution of different aerosol sources drawn through the flange without filter material. 

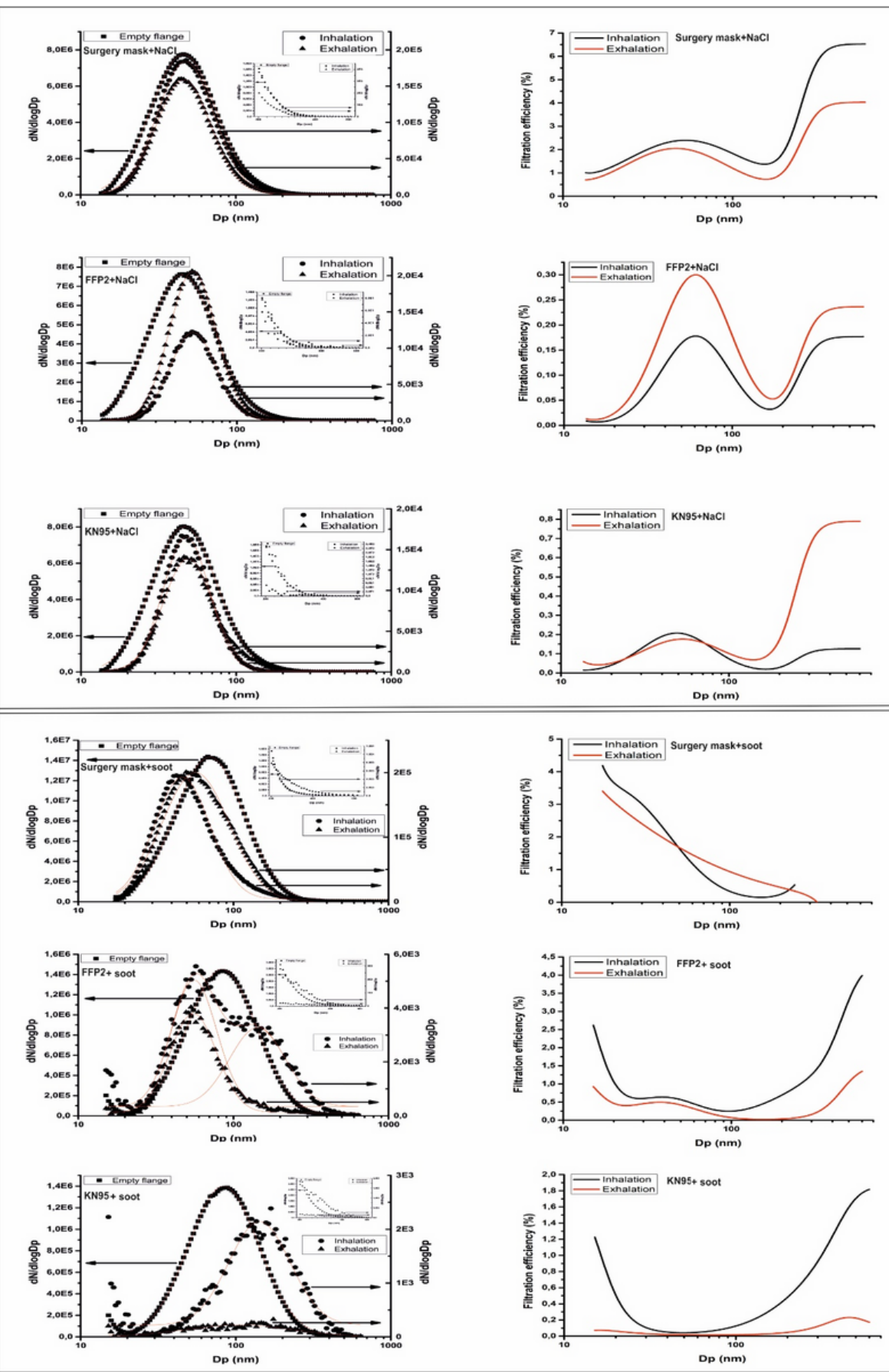

Figure 3

Measured size distribution and deduced filtration efficiency of different samples using $\mathrm{NaCl}$ and soot aerosol sources. 


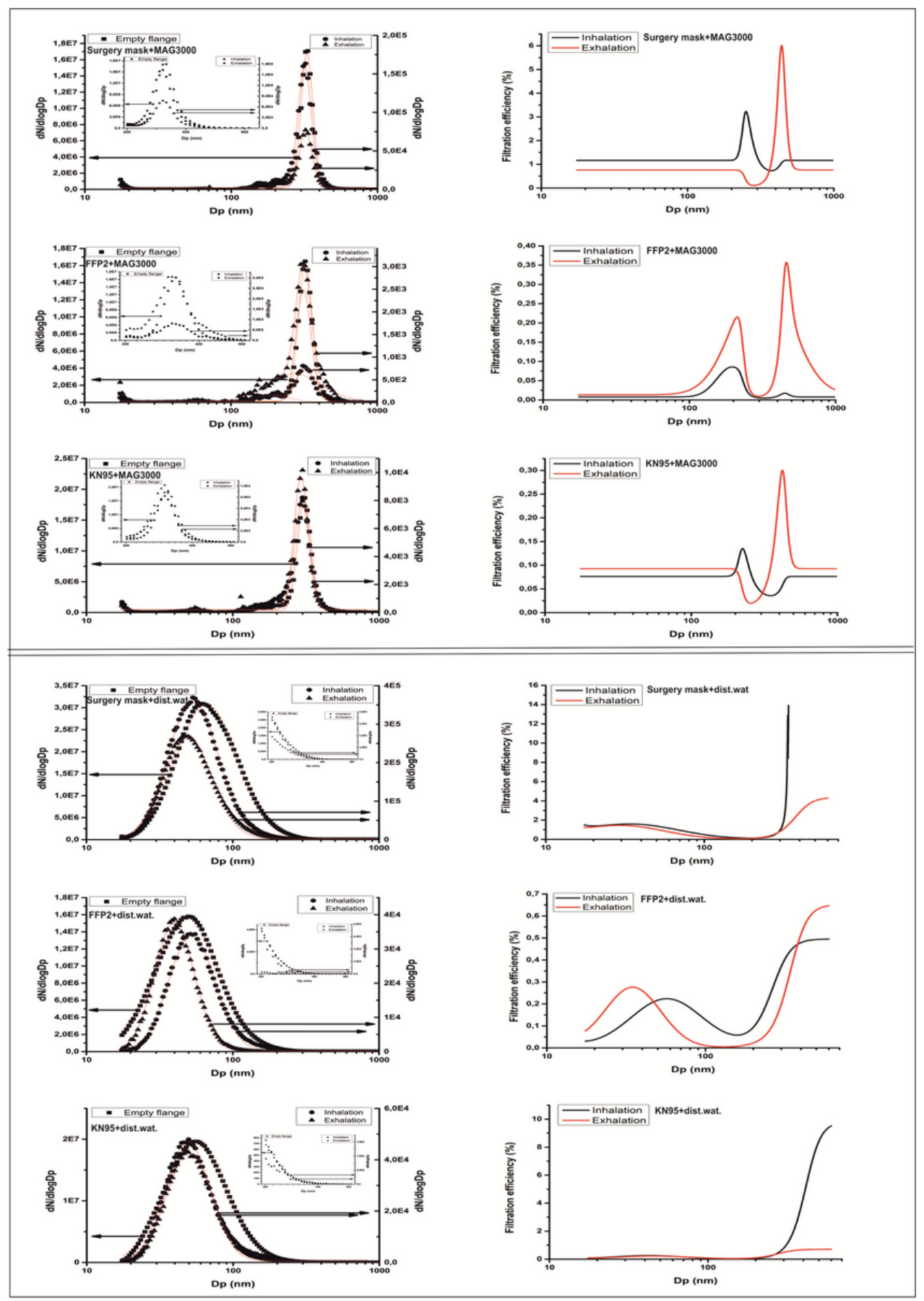

Figure 4

Measured size distribution and deduced filtration efficiency of different samples using DEHS and distilled water aerosol sources 

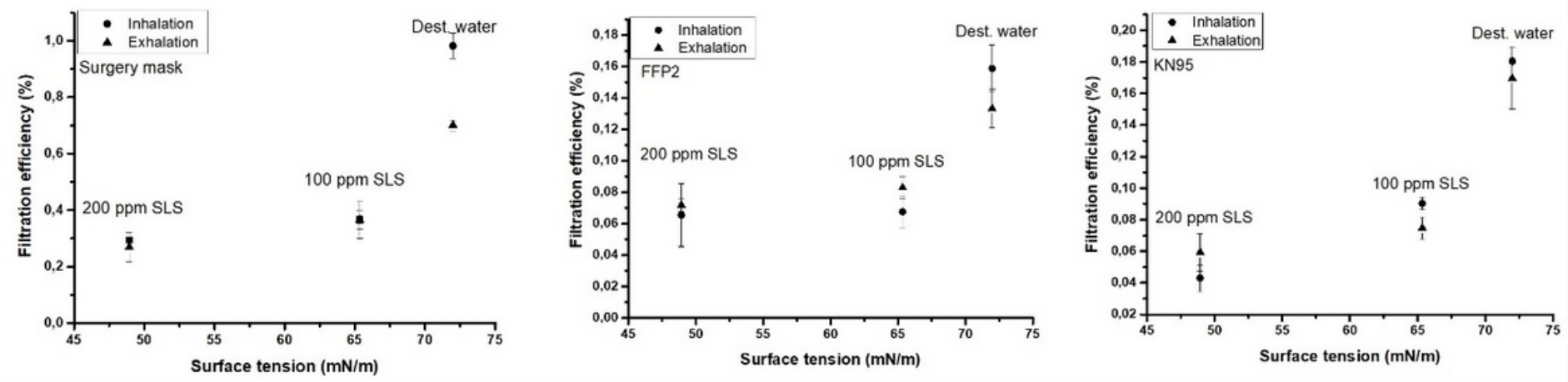

Figure 5

filtration efficiency in the function of surface tension measured on different samples.

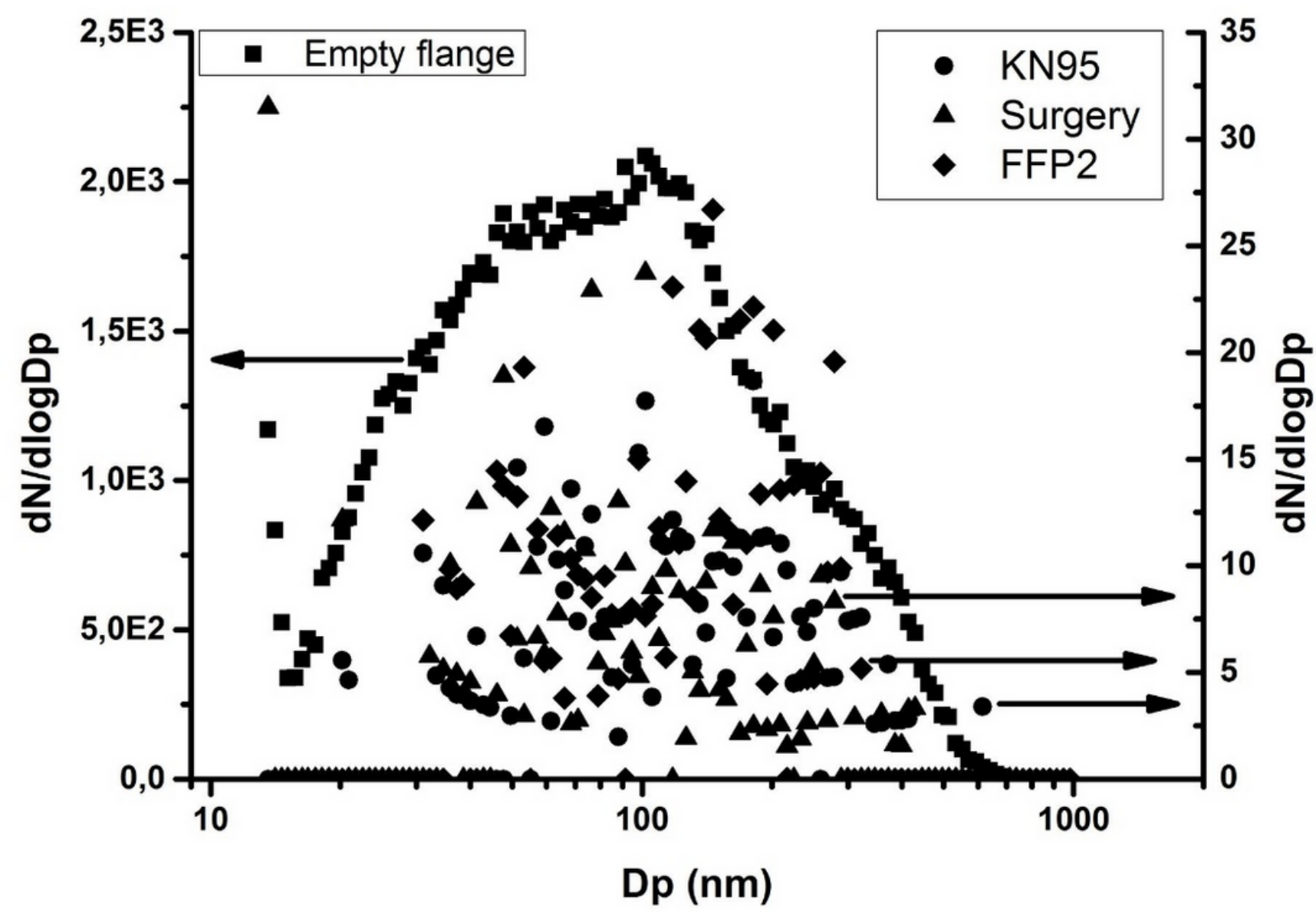

Figure 6

Measured size distribution of different samples for airborne aerosols. 